\title{
Los modelos de enseñanza de los problemas de aligación en las enciclopedias escolares españolas
}

\section{Teaching models of alligation problems in the Spanish school encyclopedias}

\author{
María Santágueda-Villanueva* \\ ORCID iD 0000-0002-5472-7972 \\ Bernardo Gómez ${ }^{* *}$ \\ ORCID iD 0000-0003-1098-4130
}

\begin{abstract}
Resumen
Los problemas de aligación forman parte de las matemáticas desde épocas antiguas. Actualmente, han perdido interés educativo, aunque siguen presentes en los temas de álgebra y en la vida diaria. En este trabajo, presentamos el modelo de enseñanza de estos problemas en las enciclopedias escolares que estuvieron vigentes desde los años veinte hasta los años setenta del pasado siglo. Para poder caracterizar el modelo de enseñanza nos fijamos en los aspectos contextuales, los estructurales, los procedimentales y los organizativos de los problemas de aligación. Realizamos una clasificación de los tipos de problemas, de los contextos utilizados, de los métodos de resolución que se utilizaban y cómo se organizaban las lecciones.
\end{abstract}

Palabras clave: Didáctica. Matemáticas. Aligación. Modelos de enseñanza. Enciclopedias.

\begin{abstract}
Alligation problems are part of mathematics since ancient times. Currently they have lost educational value, although they are still present in algebra issues and in daily life. In this paper, we present the teaching model of these problems in school encyclopaedias that were used from the twenties to the seventies of the last century. We characterize the teaching model and we look at the contextual, structural, procedural, and organizational aspects of the alligation problems. We classify the types of problems, the contexts used, the resolution methods and lesson organization.
\end{abstract}

Keywords: Didactics. Mathematics. Alloy. Models of teaching. Encyclopedias.

\section{Introducción}

La resolución de problemas es considerada una competencia básica en el desarrollo del pensamiento aritmético y algebraico (NCTM, 1980). Ejemplo de ello es que está presente en el currículo básico de la Educación Primaria española (MEC, 2006).

\footnotetext{
* Doctora en Matemática Aplicada por la Universitat de Valencia (UVEG). Profesora de Didáctica de la Matemática en la Universitat Jaume I de Castellón (UJI), Castellón de la Plana, España. E- mail: santague@uji.es. ** Doctor en Matemática por la Universitat de Valencia (UVEG). Profesor de Didáctica de la Matemática en la Universitat de Valencia (UVEG), Valencia, España. E-mail: bernardo.gomez@uv.es.
} 
La importancia de la resolución de problemas, en la educación escolar matemática, implica que el profesorado ha de conocer qué tipos de problemas hay en el currículo escolar, con qué métodos se resuelven y con qué fin se deben enseñar. Para ello, las fuentes donde el profesorado puede buscar esta información son los currículos vigentes oficiales y los libros de texto. Pero son estos últimos los más importantes ya que, como afirma Schubring (1987), para realizar una aproximación a la educación matemática de una época determinada hay que estudiar los libros escolares, ya que la práctica docente se basa en estos más que en los decretos ministeriales y los programas oficiales.

El objetivo de este trabajo es indagar sobre el modelo de enseñanza de la resolución de problemas de aligación en los años veinte hasta los años setenta del pasado siglo. Estos forman parte de la sección de proporcionalidad y son la culminación de la aritmética. El problema de aligación consiste en encontrar en qué proporción se pueden combinar dos o más cantidades, cuya combinación puede tener un valor medio o promedio.

Para delimitar el estudio, se ha escogido las enciclopedias escolares, porque fueron los libros de texto más utilizados desde los años veinte hasta los años setenta del pasado siglo, por ser muy económicos, por su estructura cíclica y graduada que se adaptaba al sistema y porque su contenido se adecuaba a las leyes educativas vigentes.

Para conseguir este objetivo se ha realizado una investigación cualitativa, exploratoria, descriptiva, cuantitativa y conceptual (FOX, 1981) y que toma como referentes teóricos los trabajos de: Gómez (2015) por su estudio de la estructura de los problemas de aligación; Gómez y Puig (2018) por su definición de lectura analítica y Madrid et al. (2017) por su esquema de análisis de contenido. Más adelante se detallan estos referentes teóricos.

\section{Las enciclopedias escolares}

A partir de los años 20 del siglo pasado, surgió el modelo de las enciclopedias que estuvo vigente hasta los años 70, eran un tipo de manual que ofrecía en un solo volumen las materias que el escolar había de cursar en cada grado o ciclo.

Como afirman Escolano (1997) y Silvestre (2015) el éxito editorial de las enciclopedias se basó en tres factores:

1. Eran baratos, en un único libro se tenían todas las materias. Se realizaban tiradas (de varios millares casos) que abarataban los costes de producción. Muchas asociaciones de maestros se convirtieron en editores para conseguir libros más económicos (ver Figura 1 izquierda punto 3). 
2. Las enciclopedias ofrecían a los maestros y a las maestras volúmenes adaptados a cada ciclo y organizados para regular la marcha de la escuela (ver Figura 1 izquierda punto 2 y Figura 1 derecha, se observa la planificación semanal).

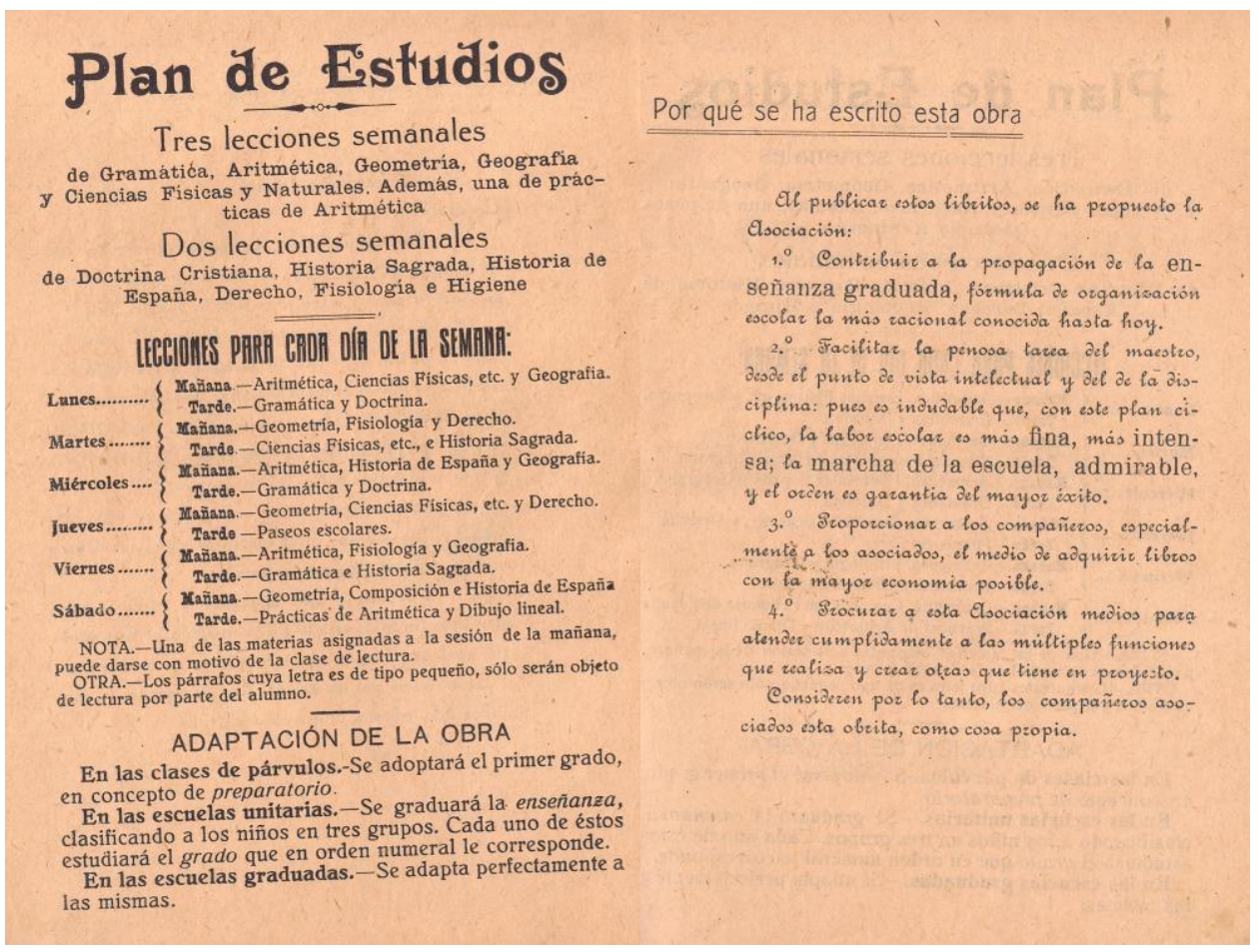

Figura 1 - Explicación del motivo de la obra

Fuente: Asociación Provincial de Maestros Nacionales (a partir de ahora APMN) (1920, p. 3)

3. Los contenidos se adecuaban a los currículos vigentes. Por ejemplo, en la Figura 2 se presenta un párrafo donde se explica que el contenido de su enciclopedia se adapta a los Cuestionarios Nacionales. 


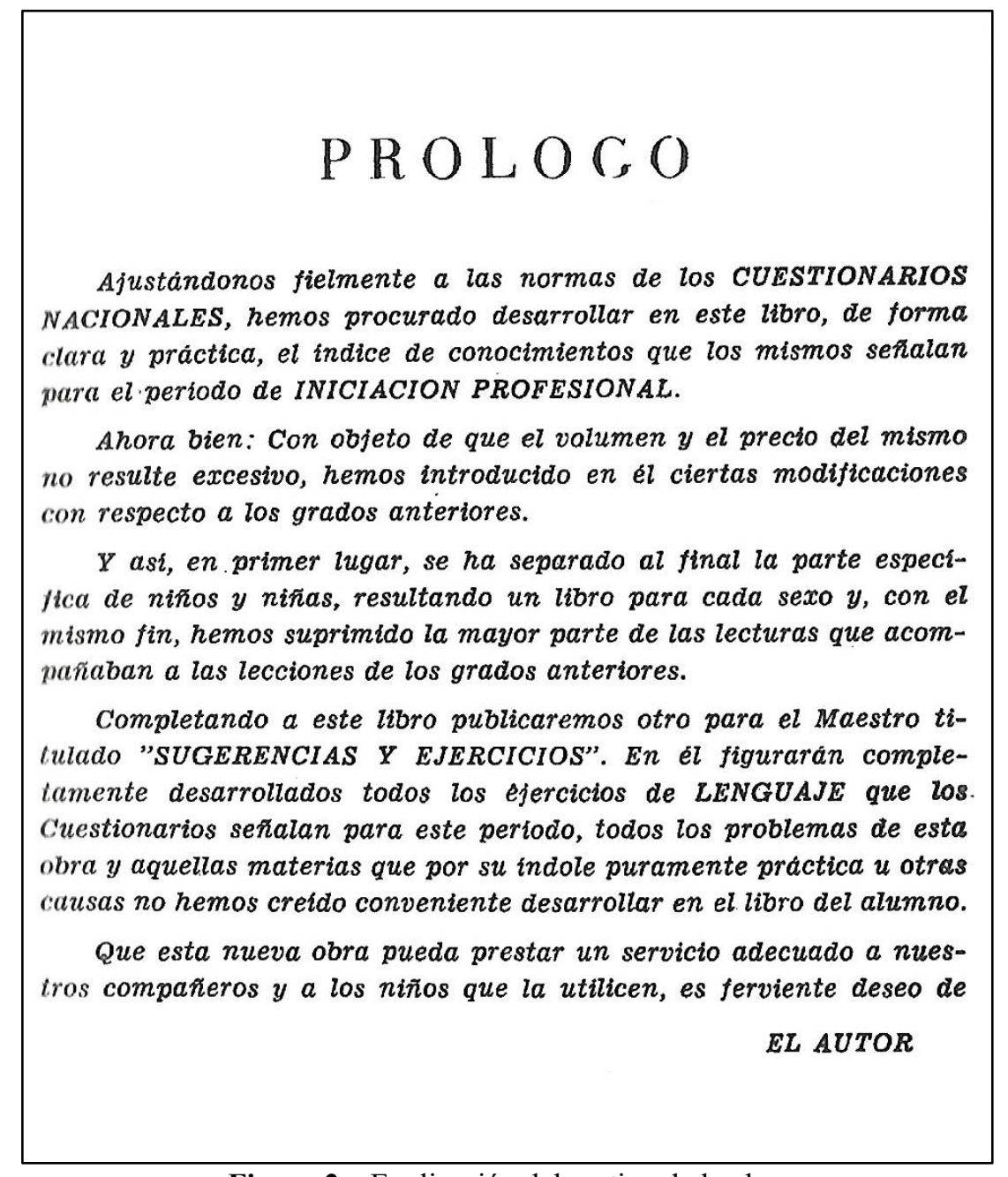

Figura 2 - Explicación del motivo de la obra Fuente: Álvarez (1971, p. 7)

Durante el uso de las enciclopedias en las escuelas hubo diversos cambios políticos y legislativos. Cabe destacar los planes de estudios de Romanones (MINISTERIO DE INSTRUCCIÓN PÚBLICA Y BELLAS ARTES, 1901) y Ruiz Jiménez (MINISTERIO DE INSTRUCCIÓN PÚBLICA Y BELLAS ARTES, 1953), por los siguientes motivos:

1. Romanones (MINISTERIO DE INSTRUCCIÓN PÚBLICA Y BELLAS ARTES, 1901) estableció la enseñanza elemental obligatoria de los 6 a los 12 años organizándola de forma cíclica en tres grados (elemental, medio y superior). Como se observa en la Figura 3, las enciclopedias se adaptan a esta graduación organizándose en tres volúmenes y cada uno contiene todas las materias que han de estudiar en ese grado. 
«El Libro de la Escuela». Enciclopedia de primera enseñanza en orden cíclico, tres grados, por la Asociación provincial de Maestros nacionales de Castellón. Consta esta obra de tres volúmenes, correspondientes, respectivamente a los grados $1^{\circ}, 2 .^{\circ}$ y $3 .^{\circ}$, conteniendo cada tomo los conocimientos que, en su grado, deben transmitirse a los escolares de las materias siguientes: Doctrina cristiana, Historia sagrada, Gramática castellana, Aritmética, Geometría, Dibujo lineal, Geografía, Historia de España, Derecho, Fisiología e Higiene y Ciencias físicas y naturales, añadiéndose en el tomo dedicado al grado $3 .{ }^{\circ}$, y a continuación de la Aritmética, el estudio del complemento de esta: asignatura, y al final del libro un buen apéndice sobre la composición. de la corteza terrestre y división y clasificación de los terrenos.

\section{Figura 3 - Explicación del motivo de la obra} Fuente: APMN (1925, p. 3)

\section{Con la ley Ruiz Jiménez (MINISTERIO DE INSTRUCCIÓN PÚBLICA Y} BELLAS ARTES, 1953) se mantuvo el orden cíclico, se reguló la enseñanza media y se generalizó la escolarización obligatoria de los 6 años hasta los 14 años, por lo que los contenidos de las enciclopedias se reorganizan y las enciclopedias se adaptaran a los cuatro grados de escolarización (primer grado, segundo grado, tercer grado e iniciación profesional) como se puede ver en la Figura 4.

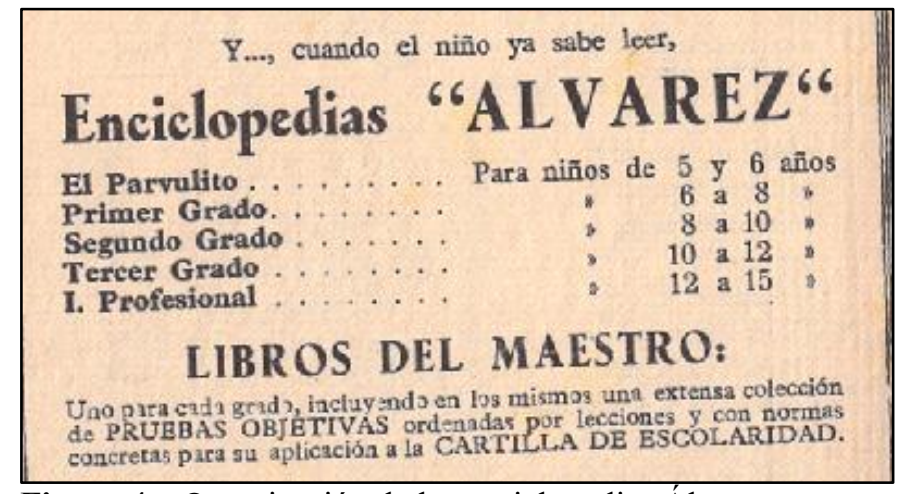

Figura 4 - Organización de las enciclopedias Álvarez Fuente: Álvarez (1953, contraportada)

A partir de la Ley Villar Palasí, MEC (1970), las enciclopedias escolares desaparecieron y se inició el uso del libro de texto que hoy conocemos, grandes series de grandes editoriales.

La manera que las enciclopedias planteaban la resolución de problemas era la siguiente: primero se enseñaban los contenidos y los procedimientos para luego resolver problemas, que coincide con una de las tres propuestas de Schroeder y Lester (1989) la enseñanza de la matemática para resolver problemas.

\section{Marco teórico}

Como fundamento teórico usamos los elementos que caracterizan los modelos de enseñanza y como fundamento metodológico las subunidades de análisis propias del análisis racional y del análisis didáctico. 


\subsection{Los modelos de enseñanza}

En este trabajo consideramos que un modelo de enseñanza es una colección de textos, con una secuencia de actividades que constituyen espacios textuales (FILLOY; ROJANO; PUIG, 2008), para generar y modelar situaciones, con un lenguaje que suele ir de lo concreto a abstracto, con códigos intermedios, para gradualmente desarrollar habilidades matemáticas y resolver distintas situaciones o problemas.

La base de un modelo de enseñanza, parte de un conocimiento matemático formal, que se transforma en actividades concretas, como un texto para producir otros textos en la interacción maestro - alumno; alumno - alumno; alumno - maestro.

Tomando esta noción como referente, usamos la expresión modelo de enseñanza aplicada a los problemas escolares, para el conjunto de textos y actividades que los contextualizan, los estructuran, los clasifican (tipos), los resuelven (métodos) y los organizan para la enseñanza. A saber:

- Contextuales: variables superficiales y circunstanciales que sitúan y adjetivan a las cantidades y describen el asunto de que trata el problema.

- Estructurales: características no circunstanciales internas, por las relaciones y conexiones entre cantidades, y externas, por sus relaciones con los conceptos y procedimientos matemáticos.

- Tipos de problemas (de la misma estructura): se refiere a las variaciones en los enunciados que son debidas a la posición de la cantidad desconocida por la que se pregunta

- Métodos de resolución: atienden a los enfoques aritmético o cartesiano para dar respuesta a la demanda del problema y se sustancian en ecuaciones, fórmulas o reglas y en la disposición práctica de los datos.

- Organizativos: son las consideraciones pedagógicas y científicas que determinan la selección y el orden de presentación y ubicación de los problemas.

\subsection{Análisis racional y análisis didáctico}

Utilizamos análisis racional y análisis didáctico, siguiendo a Lamon (2007) y a Rico, Lupiáñez y Molina (2013) respectivamente. El análisis racional para identificar los elementos 
matemáticos involucrados en los problemas y el análisis didáctico, en su dimensión de diseño e instrucción, para fijar la estructura curricular.

El análisis racional tiene por finalidad fijar los conceptos matemáticos necesarios para resolver un determinado problema. Por tanto, identifica los componentes matemáticos esenciales, tanto conceptuales como procedimentales, por lo que trata de identificar la estructura interna y externa de dicho problema.

El análisis didáctico tiene por objeto la selección y comunicación de los problemas como tareas o actividades escolares de un modelo de enseñanza en la forma en que están reflejados en los libros objeto de estudio. En este caso, son los problemas de aligación presentes en las enciclopedias escolares en un modelo de enseñanza caracterizado por los contextos, los tipos de problemas, sus métodos de resolución y su organización.

\section{Análisis racional de los problemas de aligación}

\subsection{Concepto de aligación}

Bajo el nombre de regla de aligación se conoce el procedimiento general para resolver los problemas relacionados con mezclas. Una mezcla es la unión de varias sustancias de distinta calidad y precio; si son metales se llama aleación, y si son líquidos y sustancias, como por ejemplo la mezcla de agua y sal se llama disolución. En la actualidad, parece ser que el término de aligación está en desuso y se prefiere hablar directamente de problemas de mezclas y aleaciones (ver GÓMEZ, 2015, p. 3).

Ejemplos de problemas de aligación son los siguientes, todos ellos corresponden a tipos diferentes:

P1: "Un tabernero ha mezclado 148 litros de vino a 0,50 ptas. el litro con 36 litros de 0,70 ptas. ¿A cómo resulta el precio medio?” (HIJOS DE SANTIAGO RODRÍGUEZ (H.S.R.), 1941, p. 323).

P2: "Un tabernero tiene vino de a 28 ptas. el D1., de a 26, de a 20 y de a 15 ptas. ídem, y quiere proporcionarse una quinta clase cuyo precio sea 22 ptas. el Dl. Determínese la relación de la mezcla" (DALMAU, 1958, p. 333).

P3: "Un autobús lleva 36 viajeros hasta un punto determinado. Los de primera pagaron 7,50 ptas. y los de segunda, 5 ptas. Habiéndose cobrado un total de 210 ptas., ¿cuántos viajeros iban de cada clase?" (EDELVIVES, 1957, p. 215).

P4: “¿Qué cantidad de cobre ha de añadirse a un lingote de oro de 1.800 gramos cuya ley es 0,920, para que resulte una aleación de ley 0,750?” (EDELVIVES, 1957, p. 215). 


\subsection{Estructura}

La estructura externa de los problemas de aligación es la de una composición y un promedio. La noción de composición la tomamos de Freudenthal, que se define como una terna $(\Omega, M, \omega)$, donde $\Omega$ es un conjunto de partes o clases de un universo; a cada una de las cuales se le asocia una magnitud o una medida en $M$ mediante una función $\omega$ (FREUDENTHAL, 2001). Por ejemplo, los minerales que componen una aleación forman el conjunto $\Omega$ y la función $\omega$ puede ser la que les asigna su masa (peso o porcentaje que le corresponde a la aleación). En los problemas de aligación intervienen dos funciones: $\omega_{1}$ que asigna las cantidades $m_{i}$ de cada parte o sustancia y $\omega_{2}$ que asigna los precios $p_{i}$ de cada parte o sustancia. La noción de promedio la tomamos de Wentworth (1897) quien la define como un número que en una adición se puede sustituir por los sumandos sin alterar la suma. No confundir esta definición con el algoritmo para calcularlo, $\frac{\sum \boldsymbol{m}_{\boldsymbol{i}} \boldsymbol{p}_{\boldsymbol{i}}}{\sum \boldsymbol{m}_{\boldsymbol{i}}}=\boldsymbol{P}_{\boldsymbol{m}}$, donde $\boldsymbol{P}_{\boldsymbol{m}}$ es el precio medio, que es lo que a menudo se toma como definición.

La estructura interna de los problemas de aligación viene dada por la condición esencial de toda mezcla. Esta se puede enunciar de dos maneras, una es que después de hecha la aligación debe resultar el mismo valor que por separado, o lo que es lo mismo, que el total de las cantidades ha de valer lo mismo antes y después de la mezcla, que transcrita al lenguaje algebraico es la siguiente relación de igualdad entre dos cantidades: $\boldsymbol{m}_{\mathbf{1}} \boldsymbol{p}_{\mathbf{1}}+\boldsymbol{m}_{\mathbf{2}} \boldsymbol{p}_{\mathbf{2}}=$ $\left(\boldsymbol{m}_{\mathbf{1}}+\boldsymbol{m}_{\mathbf{2}}\right) \boldsymbol{P}_{\boldsymbol{m}}(\mathbf{I}) ; \boldsymbol{m}_{\boldsymbol{i}}$ son las cantidades de cada sustancia, $\boldsymbol{p}_{\boldsymbol{i}}$ los precios y $\boldsymbol{P}_{\mathbf{m}}$ el precio medio.

Otra, es que lo que se gana con el de menor valor ha de compensar a lo que se pierde con el de mayor valor, o lo que es lo mismo, la pérdida que resulta por la mezcla de unas cantidades ha de ser igual a la ganancia que producen las otras, que transcrita al lenguaje algebraico es la siguiente relación de igualdad entre dos cantidades: $\boldsymbol{m}_{\mathbf{1}}\left(\boldsymbol{p}_{\mathbf{1}}-\boldsymbol{P}_{\boldsymbol{m}}\right)=$ $\boldsymbol{m}_{2}\left(\boldsymbol{P}_{m}-\boldsymbol{p}_{2}\right)(\mathrm{II})$.

\subsection{Tipos de problemas}

Según sea la pregunta o cantidad desconocida $\boldsymbol{P}_{\boldsymbol{m}}$ o $\boldsymbol{m}_{\mathbf{1}}$ y $\boldsymbol{m}_{\mathbf{2}}$, se conforman dos tipos principales de problemas de aligación. Los que responden a la pregunta ¿cuánto vale $\boldsymbol{P}_{\boldsymbol{m}}$ ? se denominan de aligación medial (P1), y los que responden a la pregunta ¿cuánto valen $\boldsymbol{m}_{\mathbf{1}} \mathbf{y} \boldsymbol{m}_{\mathbf{2}}$ ? se denominan de aligación alternada o inversa (P2). 
Cuando lo que se pregunta es $P_{m}$, la igualdad (I) es la que favorece la resolución, ya que al despejar la cantidad desconocida se obtiene la fórmula (III), que da la respuesta demandada: $P_{m}=\frac{m_{1} p_{1}+m_{2} p_{2}}{m_{1}+m_{2}}$

Cuando lo que se pregunta es $\boldsymbol{m}_{\mathbf{1}}$ y $\boldsymbol{m}_{\mathbf{2}}$, la igualdad que favorece la resolución es la igualdad (II), ya que al despejar se transformar en la fórmula (IV), que es la que da respuesta demandada: $\frac{\boldsymbol{m}_{1}}{\boldsymbol{m}_{2}}=\frac{\boldsymbol{P}_{\boldsymbol{m}}-\boldsymbol{p}_{2}}{\boldsymbol{p}_{1}-\boldsymbol{P}_{\boldsymbol{m}}}$ (IV)

Obsérvese que la respuesta que da la fórmula (IV) es una razón, y por tanto admite muchos valores por lo que la solución al problema es indeterminada.

Para limitar el número de respuestas hay que añadir un dato más, lo que da lugar a dos subtipos de problemas aligación alternada: la aligación alternada total (P3), donde lo que se pide son las cantidades en que entran los componentes de la mezcla conocidos los precios de las componentes, el precio medio de la mezcla y la cantidad total de la mezcla, y la aligación alternada parcial (P4), donde se pide la cantidad con que entra una de las componentes de la mezcla, sabiendo los precios de las componentes, el precio medio, y las cantidades con que entran el resto de las componentes.

Conviene señalar que no todos los problemas de mezclas son de aligación, es el caso, por ejemplo, del siguiente problema de reparto proporcional:

P5. "Para fabricar pólvora ordinaria se mezclan 3 partes de salitre, 1 parte de azufre y 1 parte de carbón de madera. ¿Qué peso de cada una de ellas hay que estas sustancias habrá que mezclar para fabricar 75 Kg. de pólvora?” (H.S.R., 1941, p. 321).

\section{Análisis didáctico de los problemas de aligación}

El análisis didáctico, comienza con la selección de las Enciclopedias que van a ser objeto de estudio. Para ello, se han escogido las de los dos niveles superiores: el tercer ciclo y el de iniciación profesional, por ser los que incluyen los problemas de aligación como parte de su contenido.

Al igual que en Madrid et al. (2017), la muestra es incidental y por conveniencia, para la elección de estas se tuvo en cuenta:

- Idioma: escritos en castellano.

- Contenidos: se trabaja los problemas de aligación.

Por tanto, de todas las enciclopedias consultadas finalmente se escogieron las que presentamos en el Cuadro 1. 


\begin{tabular}{|l|l|}
\hline Tercer grado o Perfeccionamiento & Grado Iniciación Profesional \\
\hline Dalmau (1958) & Dalmau (1959) \\
\hline H.S.R. (1941) & H.S.R. (1954) \\
\hline Edelvives (1957) & \\
\hline Álvarez (1953) & Álvarez (1971) \\
\hline
\end{tabular}

Cuadro 1 - Libros analizados.

Fuente: elaborado por el autor

El análisis didáctico continúa con la descripción de los aspectos que caracterizan el modelo de enseñanza que se desglosan en los siguientes puntos: cantidad de problemas (5.1), contextos (5.2), tipos de problemas (5.3), métodos de resolución (5.4) y la organización pedagógica (5.5). En el análisis racional se ha estudiado la estructura del problema.

\subsection{Cantidad de problemas}

En la Tabla 1 encontramos la cantidad de problemas de aligación presentes en cada enciclopedia, además de la cantidad de problemas resueltos y la cantidad de problemas que se le dejaba al alumnado para su resolución. La enciclopedia con mayor cantidad de problemas es Edelvives (1957), mientras que la enciclopedia Álvarez (1953) solamente presenta nueve problemas, de los cuales resuelve solamente uno. Se observa que los problemas resueltos varían dependiendo de la editorial y del grado al que va dirigido.

Tabla 1 - Frecuencia de problemas

\begin{tabular}{lllll}
\hline & Enciclopedia & $\begin{array}{l}\text { Total de } \\
\text { problemas }\end{array}$ & $\begin{array}{l}\text { Problemas } \\
\text { propuestos }\end{array}$ & $\begin{array}{l}\text { Problemas } \\
\text { resueltos. }\end{array}$ \\
\hline $3^{\text {o }}$ & Dalmau (1958) & 23 & 21 & 2 \\
& H.S.R. (1941) & 24 & 19 & 5 \\
& Edelvives (1957) & 66 & 61 & 5 \\
& Álvarez (1953) & 9 & 8 & 1 \\
\hline Iniciación & Dalmau (1959) & 22 & 20 & 2 \\
Profesional & H.S.R. (1954) & 18 & 11 & 7 \\
(In. Pr.) & Álvarez (1971) & 29 & 25 & 4 \\
\hline \multicolumn{4}{c}{ Fuente: elaborado por el autor }
\end{tabular}

\subsection{Contextos}

Los contextos más habituales identificados en una revisión preliminar de libros de texto de diferentes épocas, (ver GÓMEZ y SANTÁGUEDA, 2019) son: compraventa, cientificotécnico, industrial, ley y fineza para subir o bajar la calidad del oro y la plata, numerario o de cambio, ingeniosos o recreativos, de vencimiento medio o común y otros que no se pueden clasificar en estas categorías. Las enciclopedias escolares no recogen todos estos tipos, solo encontramos los siguientes: 
1. Compraventa. Situaciones de compraventa, precio, costo o intercambio de productos, bienes y artículos, con o sin beneficio para el vendedor. En estos problemas los precios son un elemento distintivo, y las cantidades habituales refieren a líquidos (vino, aceite, café, té, aguardiente etc.), cereales (arroz, maíz, trigo, harinas etc.) y otras sustancias (azúcar, algarrobas, pólvora etc.). Ejemplos ilustrativos son los dos ejemplos siguientes: P6. “Se mezclan 2461 . de vino a 0,95 ptas. el litro con 194 1. de 0,60 ptas. litro. ¿A cómo resulta el decalitro de esta mezcla?" (H.S.R., 1941, p. 326).

P7. "Un comerciante de vino mezcla 1201 . de vino de 1,15 ptas. litro con 200 litros de 1,65 ptas. ¿A cómo deberá vender el litro de la mezcla para ganar por término medio 0,35 ptas. por litro?” (H.S.R., 1941, p. 330).

Obsérvese que en el segundo ejemplo de problema comercial también se pregunta por el beneficio, si se encarece el precio de venta de la mezcla.

2. Científico-técnico. Situaciones relacionadas con la ciencia y sus tecnicismos en las que no intervienen los precios, como ocurre, por ejemplo, en las graduaciones alcohólicas, concentraciones, disoluciones etc.

P8. "Se tienen 40 litros de agua fría a $15^{\circ}$ de temperatura. ¿Qué cantidad habrá que añadirle de agua hirviendo (a $100^{\circ}$ ) para que la temperatura suba $28^{\circ}$ ?” (EDELVIVES, 1957, p. 215).

P9. “Compró una persona 1 1 1/2 litros de leche que, pura, tiene la densidad de 1,032. Habiendo pesado esta leche halló 1,540 kg. Dígase si era pura, y si no, qué proporción tenia de leche y agua" (EDELVIVES, 1957, p. 215).

3. Industrial. Situaciones donde se mezclan distintas mercancías para fabricar un nuevo producto, sin intervención de precios, como por ejemplo la obtención de tintes, jabones etc. Las cantidades habituales involucran minerales, aceites, y otras sustancias químicas. P10. "Para fabricar pólvora ordinaria se mezclan 3 partes de salitre, 1 parte de azufre y 1 parte de carbón de madera. ¿Qué peso de cada una de ellas hay que estas sustancias habrá que mezclar para fabricar 75 Kg. de pólvora?” (H.S.R., 1941, p. 321).

4. Ley y fineza para bajar o subir la calidad del oro y la plata o metales preciosos. Situaciones donde interviene metales de distintas purezas y valor, ley y peso para bajar la calidad del metal de mayor calidad. Las cantidades habituales son metales preciosos, oro y plata, y los que ligan con ellos como, por ejemplo, cobre, níquel, estaño y zinc, para hacer lo que se denomina vellón, bronce, y latón.

P11. "Un platero necesita oro de 22 quilates y sólo tiene de 18, de 23 y de 23 1/2. ¿En qué relación debe alear estas tres clases?” (DALMAU, 1958, p. 334). 
5. Numerario o cambio. Situaciones en la que interviene el cambio de monedas y que como en los casos anteriores no se hace mención a mezclas o aleaciones.

P12. "Se pagó la cantidad de 1,15 ptas. con 15 monedas, unas de 5 céntimos y otras de 10. ¿Cuántas hubo de cada clase?” (EDELVIVES, 1957, p. 215).

6. Otros. Situaciones con una supuesta aplicación práctica, en las que sin hacer mención explícita a mezclas o aleaciones se resuelven como si lo fueran.

P13. "Un autobús lleva 36 viajeros hasta un punto determinado. Los de primera pagaron 7,50 ptas. y los de segunda, 5 ptas. Habiéndose cobrado un total de 210 ptas., ¿cuántos viajeros iban de cada clase?” (EDELVIVES, 1957, p. 215).

P14. "Pesé 5 panes, sucesivamente, hallando 0.740, 0.760, 0.725, 0.746 y 0.754 kilogramos. ¿Cuál era el peso medio de un pan?” (EDELVIVES, 1957, p. 214).

Los contextos y sus frecuencias que se hemos encontrado en las enciclopedias escolares estudiadas para este trabajo son los que se recogen en la Tabla 2 . En las de $3^{\circ}$ grado el contexto más utilizado es el comercial. La enciclopedia de Edelvives (1957) es la que más cantidad de problemas presenta (66 problemas), mientras que la enciclopedia de Álvarez (1953) es la que menos (nueve problemas), todos ellos comerciales. Lo mismo sucede en las enciclopedias de iniciación profesional, donde el contexto más utilizado es el comercial.

Tabla 2 - Frecuencia relativa de los contextos.

\begin{tabular}{|c|c|c|c|c|c|c|c|}
\hline & Enciclopedia & Compraventa & $\begin{array}{l}\text { Científico- } \\
\text { técnico }\end{array}$ & Industrial. & $\begin{array}{l}\text { Oro y } \\
\text { Plata }\end{array}$ & Otros & Numerario \\
\hline \multirow[t]{4}{*}{$3^{\circ}$} & Dalmau (1958) & $15 / 23$ & $1 / 23$ & $3 / 23$ & $4 / 23$ & 0 & 0 \\
\hline & H.S.R. (1941) & $18 / 23$ & 0 & $3 / 23$ & $2 / 23$ & 0 & 0 \\
\hline & Edelvives (1957) & $28 / 53$ & $4 / 53$ & & $17 / 53$ & $2 / 53$ & $2 / 53$ \\
\hline & Álvarez (1953) & $9 / 9$ & 0 & 0 & 0 & 0 & 0 \\
\hline In. & Dalmau (1959) & $14 / 22$ & $4 / 22$ & 0 & $4 / 22$ & 0 & 0 \\
\hline \multirow[t]{2}{*}{ Pr. } & H.S.R. (1954) & $13 / 18$ & $1 / 18$ & 0 & $4 / 18$ & 0 & 0 \\
\hline & Álvarez (1971) & $25 / 29$ & $1 / 29$ & 0 & $3 / 29$ & 0 & 0 \\
\hline
\end{tabular}

Fuente: elaborado por el autor

\subsection{Tipos de problemas en las enciclopedias}

En la Tabla 3 marcamos con X los tipos de problemas que las enciclopedias ejemplifican y resuelven. En todas las enciclopedias hay al menos un problema de aligación medial resuelto, y todas, a excepción de la Álvarez (1953), presentan un problema de aligación alternada indeterminado resuelto. Por otro lado, solo Álvarez (1971) y H.S.R. $(1941,1954)$ resuelven un problema de aligación alternada total. 
Tabla 3 - Tipo de problemas explicados con ejemplo.

\begin{tabular}{llllll}
\hline & Enciclopedia & Medial & $\begin{array}{l}\text { Alternada } \\
\text { Indeterminado }\end{array}$ & $\begin{array}{l}\text { Alternada } \\
\text { total }\end{array}$ & $\begin{array}{l}\text { Alternada } \\
\text { Parcial }\end{array}$ \\
\hline $3^{\text {o }}$ & Dalmau (1958) & $\mathrm{X}$ & $\mathrm{X}$ & & \\
& H.S.R. (1941) & $\mathrm{X}$ & $\mathrm{X}$ & $\mathrm{X}$ & \\
& Edelvives (1957) & $\mathrm{X}$ & $\mathrm{X}$ & & \\
& Álvarez (1953) & $\mathrm{X}$ & & & \\
\hline In. & Dalmau (1959) & $\mathrm{X}$ & $\mathrm{X}$ & & $\mathrm{X}$ \\
Pr. & H.S.R. (1954) & $\mathrm{X}$ & $\mathrm{X}$ & $\mathrm{X}$ & $\mathrm{X}$ \\
& Álvarez (1971) & $\mathrm{X}$ & $\mathrm{X}$ & & \\
\hline
\end{tabular}

Fuente: elaborado por el autor

Como se observa en la Tabla 4 no todas las enciclopedias presentan enunciados de todos los tipos de problemas, solamente Edelvives (1957) y H.S.R. (1941) trabajan todos los tipos de problemas de aligación: medial, alternada indeterminado, alternada parcial y alternada total. Destacamos que la enciclopedia Álvarez (1953) sólo trabaja problemas de aligación medial.

Todas las enciclopedias, salvo Dalmau (1958), en los problemas de aligación medial presentan problemas de dos fases, primero se pregunta por el precio medio y luego por un beneficio, por ejemplo, tenemos el problema: “A 200 litros de vino de 2,4 pesetas le he echado 25 litros de agua. ¿A cómo sale el litro de la mezcla? Y si lo vendo a 0,35 pesetas más caro, ¿cuánto gano con la operación?” (ÁLVAREZ, 1953, p. 254). La frecuencia con que encontramos este tipo de problemas de dos fases es de: cuatro problemas en H.S.R. (1941), cinco en Edelvives (1957) y siete en Álvarez (1953).

Si contrastamos los resultados obtenidos en la Tabla 3 y en la Tabla 4 observamos que era una práctica habitual que las enciclopedias presentaran problemas de aligación de diferentes tipos, pero no se le explicaba al alumnado como se resolvían todos ellos, simplemente en la mayoría de los casos se explicaba la aligación medial, la aligación alternada indeterminada y los otros tipos se le dejaba al alumnado para su resolución.

Tabla 4 - Frecuencia relativa de los tipos de problemas encontrados

\begin{tabular}{|c|c|c|c|c|c|c|}
\hline & Enciclopedia & Medial 1 fase & Medial 2 fases & Indeterminado & parcial & Total \\
\hline \multirow[t]{4}{*}{$3^{\circ}$} & Dalmau (1958) & $6 / 23$ & 0 & $6 / 23$ & $6 / 23$ & $5 / 23$ \\
\hline & H.S.R. (1941) & $1 / 23$ & $4 / 23$ & $5 / 23$ & $2 / 23$ & $7 / 23$ \\
\hline & Edelvives (1957) & $14 / 53$ & $5 / 53$ & $10 / 53$ & $12 / 53$ & $12 / 53$ \\
\hline & Álvarez (1953) & $4 / 9$ & $5 / 9$ & 0 & 0 & 0 \\
\hline
\end{tabular}




\begin{tabular}{llllllc}
\hline In. & Dalmau (1958) & $6 / 22$ & 0 & $5 / 22$ & $6 / 22$ & $5 / 22$ \\
Pr. & H.S.R. (1954) & $5 / 18$ & 0 & $7 / 18$ & $2 / 18$ & $4 / 18$ \\
& Álvarez (1971) & $3 / 29$ & $10 / 29$ & $3 / 29$ & $5 / 29$ & $8 / 29$ \\
\hline
\end{tabular}

Fuente: elaborado por el autor

\subsection{Métodos de resolución en las enciclopedias}

A continuación, presentamos los métodos de resolución de problemas de aligación: en todas las enciclopedias estudiadas los problemas de aligación medial se resolvían de una única forma (5.4.1), lo mismo sucedía para los problemas de aligación alternada indeterminados de dos especies (5.4.2), mientras que para los problemas de aligación alternada indeterminados de tres especies o más (5.4.3) encontramos tres métodos diferentes, los cuales se aplican en parte para la resolución los problemas de aligación alternada total (5.4.4) y parcial (5.4.5).

\subsubsection{Métodos de resolución para los problemas de aligación medial}

Para el caso de aligación medial todas las enciclopedias estudiadas explican la manera de resolver los problemas de la misma forma: multiplicar la cantidad de cada producto por su precio unitario, sumar esos resultados y dividirlo por la cantidad total de la mezcla. Como ejemplo, mostramos la explicación que hace del caso Dalmau (1958) (Figura 5).

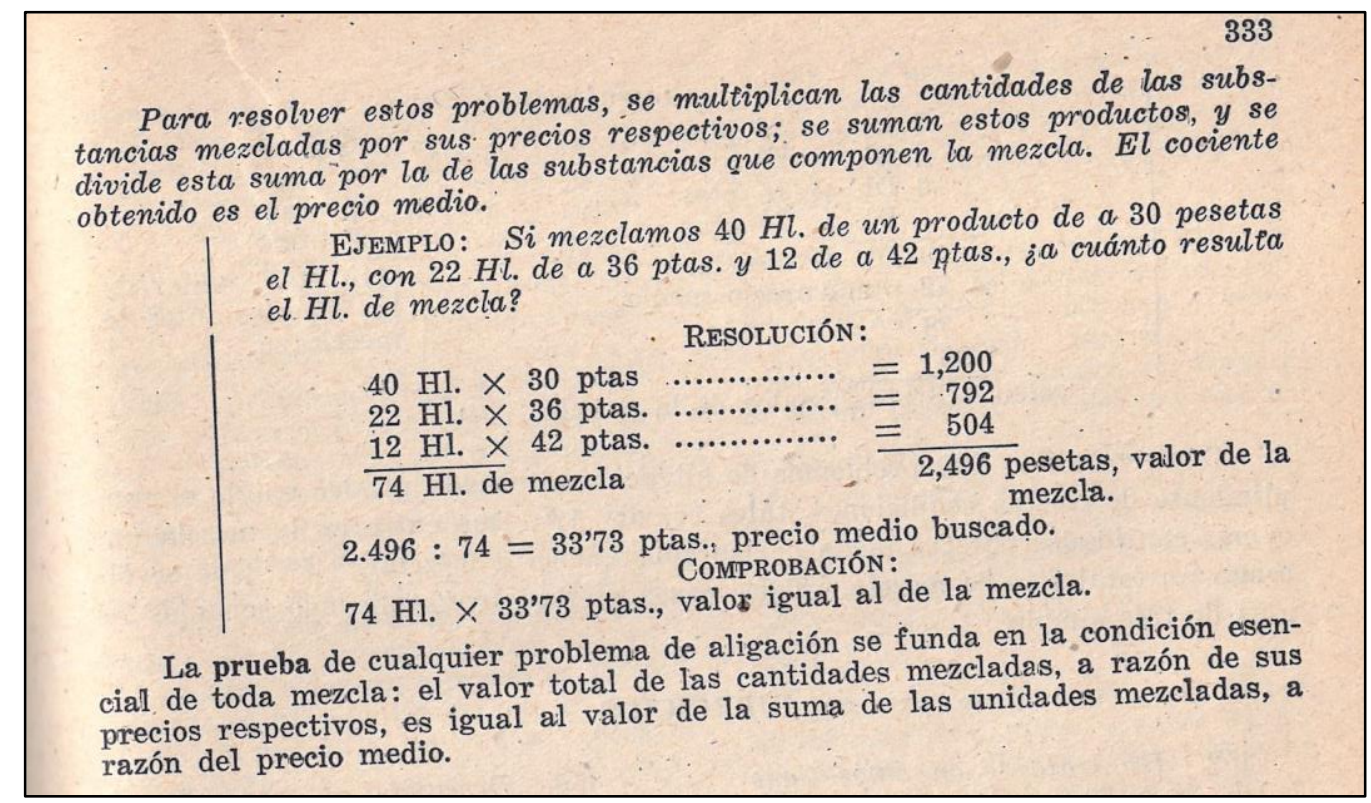

Figura 5 - Explicación y resolución de un problema medial Fuente: Dalmau (1958, p. 333) 


\subsubsection{Métodos de resolución para los problemas de aligación alternada indeterminada de dos especies}

Los problemas de aligación alternada indeterminados no son resueltos de forma algebraica, sino en la forma que se observa en la Figura 6 cuando hay dos componentes. Esta resolución se basa en la idea de lo que se gana con el de menor valor ha de compensar (ser igual) a lo que se pierde con el de mayor valor; o lo que es lo mismo, la pérdida que resulta por la mezcla de unas cantidades ha de ser igual a la ganancia que producen las otras. Lo que se reduce a la ecuación (IV). Como es bastante tedioso se suele recomendar una disposición práctica que es la que se observa en la Figura 6 y que se reduce a la fórmula que se generaliza a continuación:

$\begin{array}{lll}m_{1} & P_{m}-p_{2} \\ m_{2} & P_{m} & p_{1}-P_{m}\end{array}$

Donde $P_{m}$ el precio medio; $p_{1}$ y $p_{2}$ el precio mayor y menor; $m_{1}$ y $m_{2}$ cantidades de los precios de $p_{1}$ y $p_{2}$.

185. Cálculo de las cantidades que han de mezcrarse para
precio medio determinado. - Es el caso inverso del anterior.

IIEMPLo. - Se quiere mezclar grano de 130 ptas. el $\mathrm{Hl}$. con otro de 165 ptas.

que resulte la mezcla a 150 ptas. ¿En qué proporción se mezclarán?-El II del primer grano vale 20 ptas. menos que el de prestas diferencias. Es decir,

las. más. Se mezclarán en proporciomarán 20 del segundo.

Suele disponerse la operación en esta forma: se traza un aspa; en los iremos de la izquierda se escriben los precios dados, en el centro el precio medio que se desea; hecho lo cual, se escriben a la derecha las diferencias ntre los precios que figuran en la misma línea.

Procediendo así, se tiene frente al precio de cada susincia, la cantidad que ha de tomarse de ella.

La adjunta disposición indica que si del grano de a

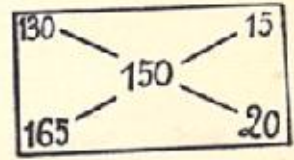

10 ptas. se toman $15 \mathrm{Hl}$, del otro han bectalitos se tomarán de uno $y$

Figura 6 - Explicación y resolución de un problema de aligación alternada indeterminado con su disposición práctica Fuente: Edelvives (1957, p. 213)

\subsubsection{Métodos de resolución para los problemas de aligación alternada indeterminada de tres o más especies}

En el caso de la aligación alternada de tres o más especies hay diferencias en el método de resolución. Como dicen en Edelvives (1957, p. 213): "El caso de haber más de dos 
sustancias. Ese caso admite un sinnúmero de soluciones". Para resolver este tipo de problemas encontramos tres métodos diferentes de resolución en las enciclopedias estudiadas, que explicamos a continuación y cuantificamos en la Tabla 5.

Tabla 5 - Presencia de los distintos métodos de resolución para los problemas de aligación alternada de tres o más especies

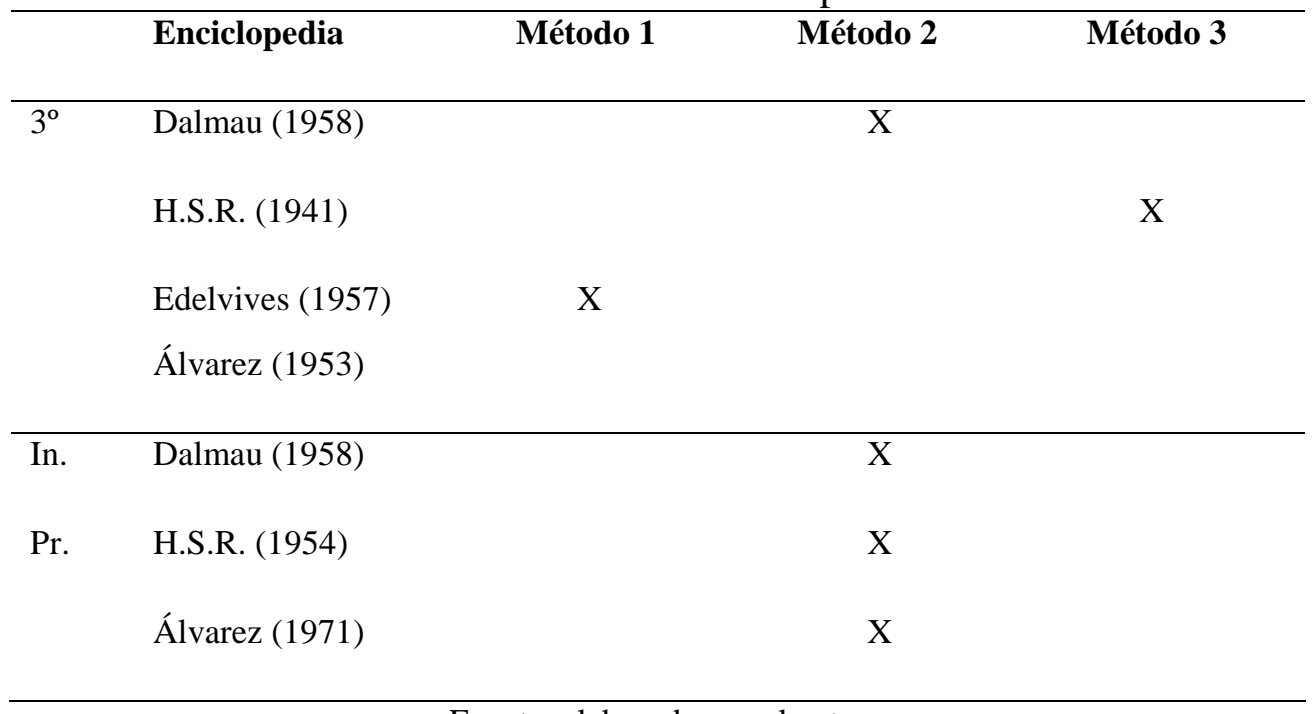

Fuente: elaborado por el autor

Método 1: en Edelvives (1957) encontramos el siguiente procedimiento: calcular las pérdidas totales (réstese el precio de cada componente cara y el precio medio, después súmese todas) y también las ganancias totales (réstese el precio de cada componente barata y el precio medio, después súmese todas). Después, de cada una de las sustancias de pérdida se ha de poner la cantidad de ganancia total, mientras que de cada una de las sustancias que hay ganancia hay que poner la cantidad de pérdida total. Este método es que sirve si hay más sustancias con las que perdemos dinero o no, ya que se reduce al caso de dos sustancias. Ver Figura 7.

186. Caso de haber más de dos sustancias. - Este caso admite un sinnúmero de soluciones. Podría procederse, vg., de modo análogo al empleado

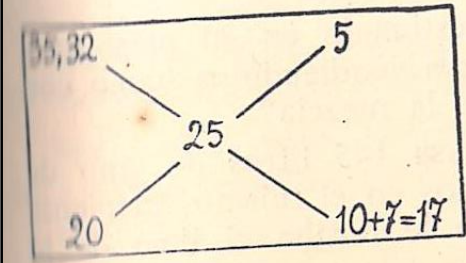
para dos sustancias. Ej.:

Con cafés de 35,32 y 20 ptas. el kilo, se desea preparar una mezcla que resulte a 25 ptas. Indicar una de las soluciones posibles. - El adjunto esquema muestra que puede resolverse el caso mezclando $5 \mathrm{Kg}$. de cada uno de los dos prime es de precio insuperior, con 17 del tercero, que es ferior.

187. Aleaciones. - Ya se vio (n. ${ }^{\circ}$ 132) lo que significa la palabra ley hablando de metales preciosos. Las mezclas de metales constituyen aleacio

Figura 7 - Explicación y resolución de un problema de aligación alternada indeterminado con tres componentes, reduciendo al caso anterior

Fuente: Edelvives (1957, p. 213) 
Método 2: lo encontramos en Dalmau (1958) y Álvarez (1971), es necesario tener el mismo número de sustancias con las que se pierda dinero y que se gane. Si esto no sucede, lo que hacen los autores es duplicar las sustancias necesarias hasta que se consiga, por ejemplo, en el caso de Edelvives (1957) (Figura 7) se tendría que escribir dos veces el 20 al ponerlo de forma esquemática. Una vez ordenados los precios de menor a mayor, o al revés, y teniendo la misma cantidad de sustancias (pierde y gana) se empieza a realizar las diferencias de forma que la que se pierde más es la cantidad que se necesita de la sustancia más cara, así sucesivamente hasta que la que más se gana es la cantidad de la sustancia que más se pierde. Ver la Figura 8.

Problema general de aligación alternada. -

Conociendo el precio medio y los precios de las especies, hallar la relación en que debe hacerse la mezcla.

Su resolución. -

Para determinar la relación en que debe hacerse una mezcla, se escriben los precios de las especies en columna y de mayor a mencribe el precio medio; se se encierran con una llave, enfrente de la cual se menor; se resta cada uno toman dos precios, uno mayor que el med invertidas. Estas diferencias indican

la relación en que debe hacerse la mezcla.

EJEMPLo. - Un tabernero tiene vino de a 28 ptas. el $D l$., de a 26, de a 20 y de a 15 ptas. idem, y quiere proporcionarse una quinta clase cuyo precio sea 22 ptas. el $D l$. Determínese la relación de la mezcla.

\section{RESOLUCIÓ:}

$$
\begin{aligned}
& \begin{array}{lllllll}
28 & \text { ptas. } \\
26 & \ldots & \ldots & \ldots & \ldots & \ldots & 22-15=7 \\
\ldots & \ldots & \ldots & \ldots & 22-20=2
\end{array} \\
& 22 \text { ptas. }
\end{aligned}
$$

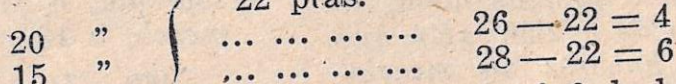

15 …........ tomará 6 de la $4 .^{a}$, y por cada

Por cada $7 \mathrm{Dl}$. de la $1 .{ }^{\mathrm{a}}$ clase,

Figura 8 - Explicación y resolución de un problema de aligación alternada de cuatro componentes, organizando las componentes de mayor a menor y reduciendo al caso de dos componentes

Fuente: Dalmau (1958, p. 333)

Método 3: H.S.R. (1941) intenta reducir el problema a un problema más sencillo. La forma escogida es hacer parejas de dos en dos, de forma que siempre se cogen dos elementos donde uno tiene el precio superior al de la mezcla requerida y el otro es inferior (ver Figura 9). En H.S.R. (1941, p. 324) para el caso de que el número de precios o calidades es impar, se dice textualmente: "A veces, el número de precios o calidades es impar, o sin ser impar no pueden combinarse de dos en dos, uno mayor y otro menor que el precio medio. En este caso, se toma un precio más de una vez para que siempre haya uno mayor y otro menor que el precio medio". Además, nos dan un ejemplo en las Figuras 9 y 10. 
4. Cuando son más de dos las especies que han de mezclarse.-Cuando son más de dos los precios o especies que han de mezclarse, se reduce este caso al anterior combinando los precios de dos en dos con, el precio medio, cuidando de que los precios sean siempre uno mayor y otro menor que el precio medio.

Ejemplo:

Mezclemos café de 12 ptas., de 10 ptas., de 8 ptas. $y$ de 7 ptas. kilogramo. para obtener una cantidad que valga a 9 ptas. kilogramo. ¿Cuántos kilogramos tomaremos de cada precio?

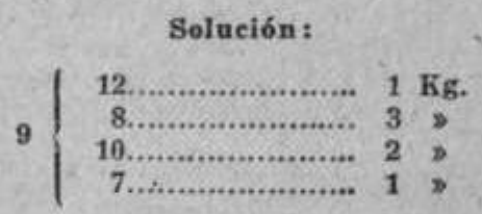

Figura 9 - Explicación y resolución de un problema de aligación alternada de cuatro componentes (dos especies son más caras que el precio que se pide y dos son más económicas), el método de resolución es haciendo parejas Fuente H.S.R. (1941, p. 324)

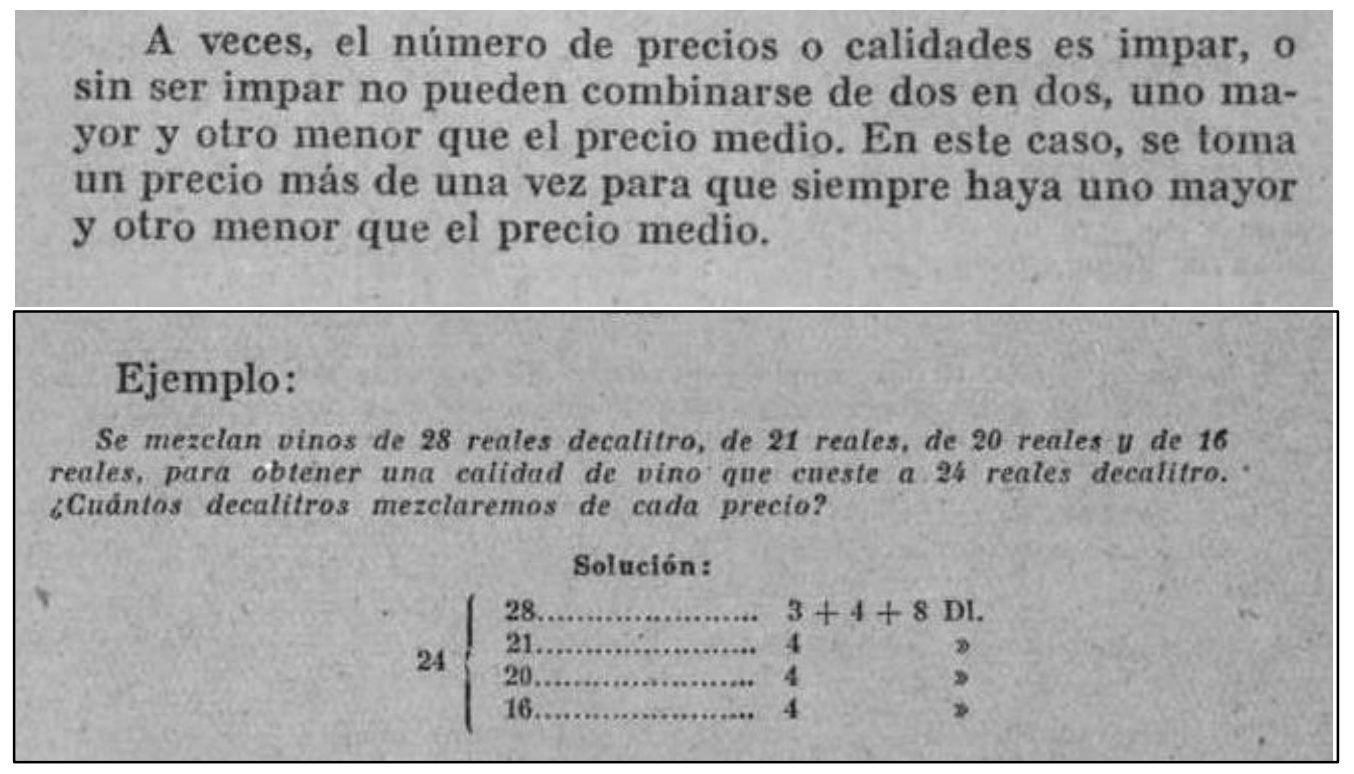

Figura 10 - Explicación y resolución de un problema de aligación alternada de cuatro componentes (una especie es más caras que el precio que se pide y tres son más económicas), el método de resolución es haciendo parejas Fuente: H.S.R. (1941, p. 324-325)

\subsubsection{Métodos de resolución para los problemas de aligación alternada total}

En el caso de la aligación alternada total solo lo encontramos en Dalmau (1958, 1959), la H.S.R. (1941, 1954) y Álvarez (1971). Por ejemplo, en H.S.R. (1941, p. 325) se dice: "Cuando el problema se da la suma de las cantidades que han de mezclarse, además de los precios medios y los precios de distintas especies o calidades, se divide dicha suma en partes proporcionales a las diferencias que indican la relación de las cantidades que se mezclan.” 
Además, presenta un ejemplo, ver la Figura 11, el autor primero resuelve el problema como si fuera un problema de aligación alternada indeterminada y, después, para poder calcular la cantidad que necesita de cada especie para elaborar la mezcla pedida, utiliza las razones de proporción, algunos autores utilizan la fórmula y otros directamente la regla.

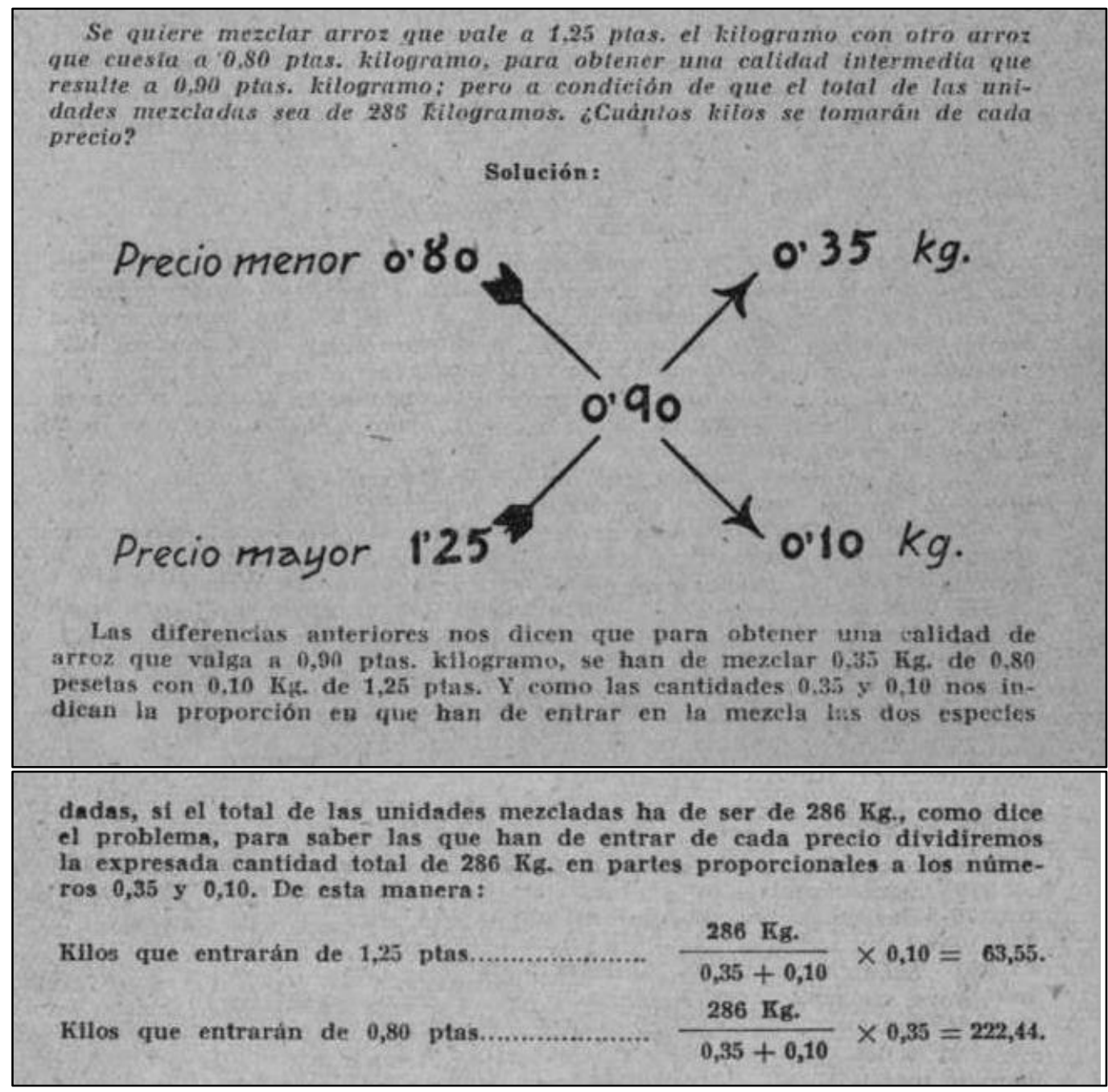

Figura 11 - Explicación y resolución de un problema de aligación alternada total con dos especies, método de resolución utilizando razones de proporción Fuente: H.S.R. (1941, p. 325-326)

Tanto Álvarez (1971) como Edelvives (1957) tienen una lección dedicada a resolución de problemas con ecuaciones y en ambos encontramos que en el listado que se le ofrece al alumnado para practicar lo aprendido, hay un problema de aligación alternada total en cada una de las enciclopedias para resolverlos con este método, pero no está resuelto.

\subsubsection{Métodos de resolución para los problemas de aligación alternada parcial}

Para la aligación alternada parcial Álvarez (1971) lo divide en dos casos, el primero es que, además del precio medio y cantidad total, se conoce la cantidad de una sustancia que forma, 
y el segundo, que conozcamos más de una de las cantidades que forman la mezcla, la resolución del segundo caso se reduce al primero ya que se ha de calcular el precio medio de las cantidades conocidas y la cantidad de producto que ya se tiene.

Para el primer caso, la propuesta que hace Álvarez (1971) para la resolución de un problema de aligación alternada parcial de tres componentes, conociendo la cantidad de una especie que forma la mezcla, es primero se resuelve el problema como si fuera un problema de aligación alternada indeterminada y cuando se conoce las proporciones, se pasa a calcular la cantidad de sustancias de cada elemento utilizando la regla de tres con su disposición práctica (Figura 12).

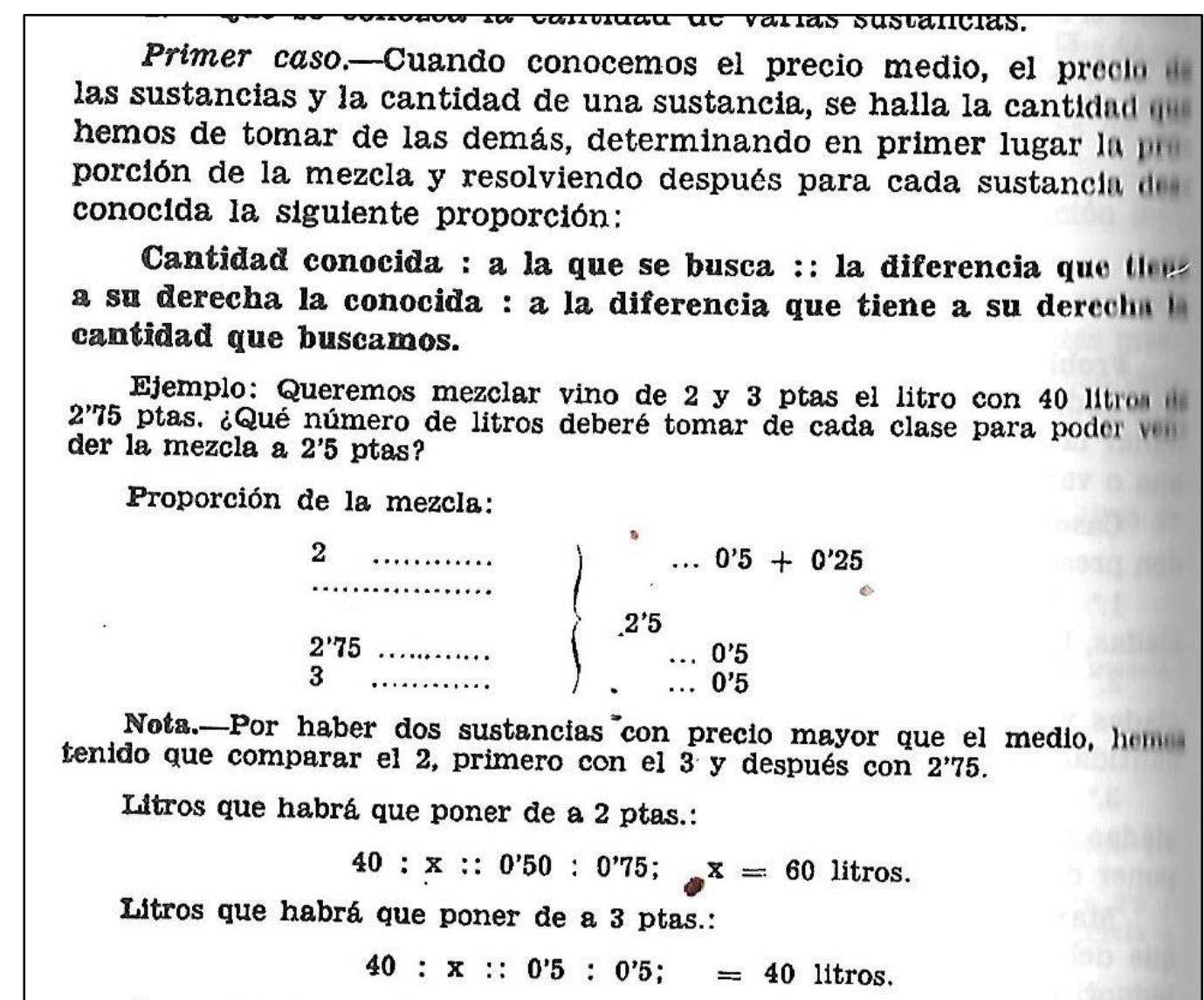

Figura 12 - Explicación y resolución de un problema de aligación alternada parcial de tres componentes conociendo la cantidad de una especie que forma la mezcla, método de resolución utilizado es la regla de tres con su disposición práctica Fuente: Álvarez (1971, p. 396)

\subsection{Organización pedagógica}

Hay que recordar al lector que las enciclopedias escolares se dividían por lecciones y se utilizaban durante dos cursos, los problemas de aligación era un contenido de las enciclopedias de tercer grado (último curso de Romanones y penúltimo en Ruiz Jiménez) o de iniciación profesional (último curso de Ruiz Jiménez) y se encontraban en las últimas lecciones. Hay que 
destacar que todas las enciclopedias tenían una lección específica dedicada a este contenido. Además, las enciclopedias Edelvives (1957) y la H.S.R. (1941, 1954) al finalizar las lecciones presentan una cantidad más o menos extensa de problemas de recapitulación, donde también encontramos problemas de aligación.

Además, en la enciclopedia Edelvives (1957) observamos que en la lección de aligación distingue entre los problemas de mezclas y los de aleaciones.

La estructura de la lección era igual en todas las enciclopedias, primero se le daba al alumno un listado de definiciones, luego se resolvían unos problemas y, por último, de forma más o menos extensa, habían unos problemas para practicar, que coincide con una de las tres propuestas de Schroeder y Lester (1989): la enseñanza de la matemática para resolver problemas.

\section{Conclusiones}

En el presente trabajo hemos caracterizado los modelos de enseñanza para los problemas escolares por su contextualización, su estructura, por los tipos de problemas que encontramos, los métodos de resolución y los aspectos organizativos. Posteriormente, lo hemos aplicado en el estudio de los problemas de aligación en las enciclopedias escolares. Como conclusión destacamos que:

1. Los problemas de aligación están presentes en todas las enciclopedias de tercer grado en sus últimas lecciones. La enciclopedia Álvarez (1953) solo presenta la aligación medial, pero en su enciclopedia de iniciación profesional de esta editorial (ÁLVAREZ, 1971) encontramos todos los tipos de aligación.

2. Los contextos son variados, aunque los más presentes son el de compraventa, del de oro y plata, el industrial y el científico-técnico. La enciclopedia Edelvives (1957) es la que más contextos presenta, también es la que más cantidad de problemas tiene.

3. En las enciclopedias escolares no existe un único modelo de resolución de los problemas de aligación alternada indeterminada cuando la mezcla se compone de tres especies o más, sección 5.4.3. Por ejemplo, algunas enciclopedias hacen parejas para reducirlo al caso sencillo, otras proponen ordenar el precio de las especies para luego generalizar el caso de dos especies y otras simplemente calculan beneficios y pérdidas. Todas las enciclopedias evitan la resolución algebraica, aunque Álvarez (1971) y Edelvives (1957) presentan un problema de aligación alternada total, en el listado de problemas para resolver, utilizando ecuaciones en una sección de ampliación. 
4. Para los problemas de aligación alternada total aplican el método explicado para la aligación alternada indeterminada y lo complementan con reglas de tres. Mientras que los problemas de aligación alternada parcial no son explicados en la mayoría de los textos, Álvarez (1971) sí que lo hace y distingue varios casos.

Podemos concluir que con este estudio mostramos que el razonamiento aritmético utilizado para resolver los problemas de aligación alternada es, en sí mismo, una rica fuente de conocimiento. Por lo tanto, puede ser útil enseñar trabajar estos problemas como objeto de estudio en lugar de encontrar su solución como subproducto de otra rama del aprendizaje, como el álgebra. Nuestro desafío es mantener vivo este conocimiento, evitando que sea olvidado.

\section{Agradecimientos}

Este trabajo ha contado con el apoyo del proyecto concedido por el Ministerio de Educación de España EDU2017-84377-R.

\section{Referencias}

ÁLVAREZ. Enciclopedia intuitiva, sintética y práctica: Tercer grado. Valladolid: Miñón, 1953.

ÁLVAREZ. Enciclopedia intuitiva, sintética y práctica: Iniciación profesional. Valladolid: Miñón, 1971.

ASOCIACIÓN PROVINCIAL DE MAESTROS NACIONALES (APMN). EI libro de la escuela: enciclopedia de primera enseñanza de orden cíclico - tercer grado. Castellón: Benjamín Ballester, 1920.

ASOCIACIÓN PROVINCIAL DE MAESTROS NACIONALES (APMN). El libro de la escuela: enciclopedia de primera enseñanza de orden cíclico - segundo grado. Castellón: Benjamín Ballester, 1925.

DALMAU. Enciclopedia Estudio Libro Azul. Girona: Carles Dalmau Pla, 1958.

DALMAU. Enciclopedia Estudio Grado de Iniciación Profesional Industrial y Artesanía. Girona: Carles Dalmau Pla, 1959.

EDELVIVES Enciclopedia tercer grado. Zaragoza: Luis Vives, 1957.

ESCOLANO, A. Historia ilustrada del libro escolar en España: Del Antiguo Régimen a la Segunda República. Madrid: Fundación Germán Sánchez Ruipérez, 1997.

FILLOY, E.; ROJANO, T.; PUIG, L. Educational Algebra: A Theoretical and Empirical Approach. New York: Springer, 2008.

FOX, D. J. El proceso de investigación en educación. Pamplona: Universidad de Navarra, 1981.

FREUDENTHAL, H. Fenomenología didáctica de las estructuras matemáticas. Traducción Luis 
Puig. Ciudad de México: Dpto de Matemática Educativa/CINVESTAV, 2001.

GÓMEZ, B. Los problemas de aligación. XIV CIAEM-IEACME (Conferencia Iberoamericana de Educación Matemática). Chiapas: Tuxtla Gutierrez, 2005.

GÓMEZ, B.; PUIG, L. Oh tú que indicas tan bien las horas, ¿cuántas han pasado desde esta mañana? Problemas descriptivos de fracciones. En: FLORES, P.; LUPIÁÑEZ, J.; SEGOVIA I. Enseñar Matemáticas. Homenaje a los profesores Francisco Fernández y Francisco Ruiz. Granada: Atrio, 2018. p. 115-128.

GÓMEZ, B.; SANTÁGUEDA, M. Elementos de los modelos de enseñanza de los problemas de aligación: contextos, estructura y tipos. En: CODINA A.; MORENO, M. F. (Eds.) Investigaciones en Pensamiento Numérico y Algebraico: Homenaje a Francisco Gil Cuadra. No2 (Colección Ciencias Humanas y Sociales). Almería: Editorial de la Universidad de Almería, 2019.

HIJOS DE SANTIAGO RODRÍGUEZ (H.S.R.). Nueva enciclopedia escolar: Grado tercero. Burgos: Hijos de Santiago Rodríguez, 1941.

HIJOS DE SANTIAGO RODRÍGUEZ (H.S.R.). Nueva enciclopedia escolar: Iniciación profesional. Burgos: Hijos de Santiago Rodríguez, 1954.

LAMON, S. J. Rational numbers and proportional reasoning: Towards a theoretical framework for research. En: LESTER, F. K. (ed.). Second handbook of research on mathematics teaching and learning. Charlotte: Information Age Pub., 2007. p. 629-667.

MADRID, M. J.; MAZ-MACHADO, A.; LEÓN-MANTERO, C.; LÓPEZ-ESTEBAN, C. Aplicaciones de las Matemáticas a la Vida Diaria en los Libros de Aritmética Españoles del Siglo XVI. Bolema, Rio Claro, v. 31, n. 59, p. 1081-1100, 2017.

MINISTERIO DE EDUCACIÓN Y CIENCIA. Ley 14/1970, de 4 de agosto, General de Educación y Financiamiento de la Reforma Educativa. Boletín Oficial del Estado (BOE), Madrid, n. 187, p. 12525-12546, 1970.

MINISTERIO DE EDUCACIÓN Y CIENCIA. Ley Orgánica 2/2006, de 3 de mayo, de Educación. Boletín Oficial del Estado (BOE), Madrid, n. 106, p. 17158-17207, 2006.

MINISTERIO DE INSTRUCCIÓN PÚBLICA Y BELLAS ARTES. Real Decreto de 19 de agosto Gaceta de Madrir (GM), Madrid, n. 231 p. 790-795, 1953.

MINISTERIO DE INSTRUCCIÓN PÚBLICA Y BELLAS ARTES. Ley de 26 de febrero, sobre Ordenación de Enseñanzas Medias. Boletín Oficial del Estado (BOE), Madrid, n. 58 p. 1119-11130, 1953.

NCTM. An Agenda for Action: recommendations for school Mathematics of the 1980s. Reston: National Council of Teachers of Mathematics, 1980.

RICO, L.; LUPIÁÑEZ, J. L.; MOLINA, M. Análisis Didáctico en Educación Matemática: Metodología de investigación, formación de profesores e innovación curricular. Granada: Comares, 2013.

SCHROEDER, T.L.; LESTER, F.K., Jr. Developing understanding in mathematics via problem solving. En: TRAFTON, P.R.; SHULTE, A.P. (ed.). New directions for elementary school mathematics. Reston, VA: National Council of Teachers of Mathematics, 1989. p. 31-42.

SCHUBRING, G. On the methodology of Analysing Historical Textbooks: Lacroix as textbook 
author. For the Learning of Mathematics, New Westminster, BC, v. 7, n. 3, p. 41-51, 1987.

SILVESTRE, M. D. S. La didáctica de la lengua española en los «Manuales Enciclopédicos Escolares» del grado o nivel elemental de educación primaria (1845-1970). 2015. Tesis

(Doctorado en Didáctica de la Lengua y la Literatura) - Universidad de La Rioja, Logroño, 2015.

WENTWORTH, G. Aritmética práctica. Boston: Ginn y compañia, 1897.

Submetido em 30 de Janeiro de 2020. Aprovado em 12 de Agosto de 2020. 